\section{Congenital retroperitoneal large size neuroblastoma} Address for correspondence: Neveen Shalalfa,
Epidemiologist, Family Medicine Specialist, Ministry of
Health, Palestine. Email: noviheeh@gmail.com

How to cite this article: Amro W, Shaltaf A, Shalalfa N. Congenital retroperitoneal large size neuroblastoma. G Med Sci. 2021; 2(2): 001-003.

https://www.doi.org/10.46766/thegms.pedia.21030202

\author{
Wael Amro ${ }^{1}$, Ahmad Shaltaf ${ }^{1}$, Neveen Shalalfa ${ }^{2 *}$ \\ 'Pediatric Surgeon, Ministry of Health, Palestine \\ ${ }^{2}$ Epidemiologist, Family Medicine Specialist, Ministry of Health, Palestine
}

Copyright: @ 2021 Wael Amro, Ahmad Shaltaf, Neveen Shalalfa. This is an Open Access article distributed under the Creative Commons Attribution License, which permits unrestricted use, distribution, and reproduction in any medium, provided the original work is properly cited.

\begin{abstract}
We experienced a rare case of 9-months-old girl with an abdominal neuroblastoma that exhibited radiographic evidence of tumor extension into themesenteric artery. Imaging studies were suggestive of neuroblastoma which also confirmed by the histologic analysis that show a diagnosis of neuroblastoma too. The patient never underwent initial chemotherapy for the treatment of neuroblastoma but experienced operation for tumor and resection was successfully achieved. This case and the treatment strategy are discussed in this paper.
\end{abstract}

Keywords: Neuroblastoma, Pediatric surgery, Chemotherapy

\section{Introduction}

Neuroblastoma is an embryonal malignant tumor developing from the precursor cells of the sympathetic nervous system. It constitutes $8-10 \%$ of all malignancies in children aged under 15 ( $7.8 \%$ in the USA, $9 \%$ in Germany), and in the structure of malignant extracranial tumors of childhood, it is ranked first, ahead of leukemias [1].

Each year, about 800 children age 0 to 14 are diagnosed with neuroblastoma in the United States. It accounts for $6 \%$ of all childhood cancers in the United States. Almost $90 \%$ of neuroblastoma is found in children younger than 5. It is the third most common type of cancer in children in general, with the average age of diagnosis between 1 and 2 of age. The disease is the most commonly diagnosed cancer in children younger than one year [7].

Neuroblastomas tumors are one of the most common abdominal solid tumors in children. The differential diagnosis of these tumors should be made based on the findings of biopsies, tumor markers and imaging such as ultrasonography and computed tomographic scanning in order to facilitate clinical decision-making [2].
However, most tumors are occult and rapidly metastasize throughout the body, ultimately becoming life-threatening. Surgical resection is an effective treatment for children. Minimally invasive surgery has unique advantages in the resection [3].

Cases of pediatric neuroblastoma presenting with intracaval and intracardiac tumor extension can be treated appropriately with initial chemotherapy followed by surgical resection of the primary and extended tumors. However, surgery to remove the primary tumor should be considered [2].

\section{Case report}

A 9-month-old girl, O+ blood group, was admitted to our hospital complaining of an abdominal mass which was discovered by her parents. On physical examination, her vital signs were within the normal limits, she was up-to-date of her vaccination with no pervious history of any operation or known disease. Her laboratory tests of serum Potassium was $4.9 \mathrm{mmol}$, Sodium $134 \mathrm{mmol}$, Calcium $11.6 \mathrm{mg} / \mathrm{dl}$, Chloride $102 \mathrm{mEq} / \mathrm{l}$, Creatinine $0.3 \mathrm{mg} / \mathrm{dl}$, and Hemoglobin $11.4 \mathrm{~g} / \mathrm{dl}$. The prothrombin time, and all coagulation tests were normal. 
However, a right-sided abdominal mass extending to the midline was palpated. The findings included elevated levels of aspartate aminotransferase (AST) $67 \mathrm{U} / \mathrm{L}$, lactate dehydrogenase (LDH) also was elevated. Abdominal ultrasonography and enhanced computed tomographic scanning revealed a large mass in the retroperitoneal cavity in sub hepatic region with multiple internal foci of multiple calcification, the mass was measured about $8.7^{*} 8.3 \mathrm{~cm}$ (Fig. 1). Based on these images, the tumor was initially considered to be at Stage 3. Chemotherapy was not given and operation was done successfully for tumor as it was operable, and then sent for pathology report.

The pathology report revealed neuroblastoma with low mitotic karyorrhectic index and no Schwan stroma. On discharge the girl was well, active, and with stable vital signs. She was able to tolerate her regular feeding.

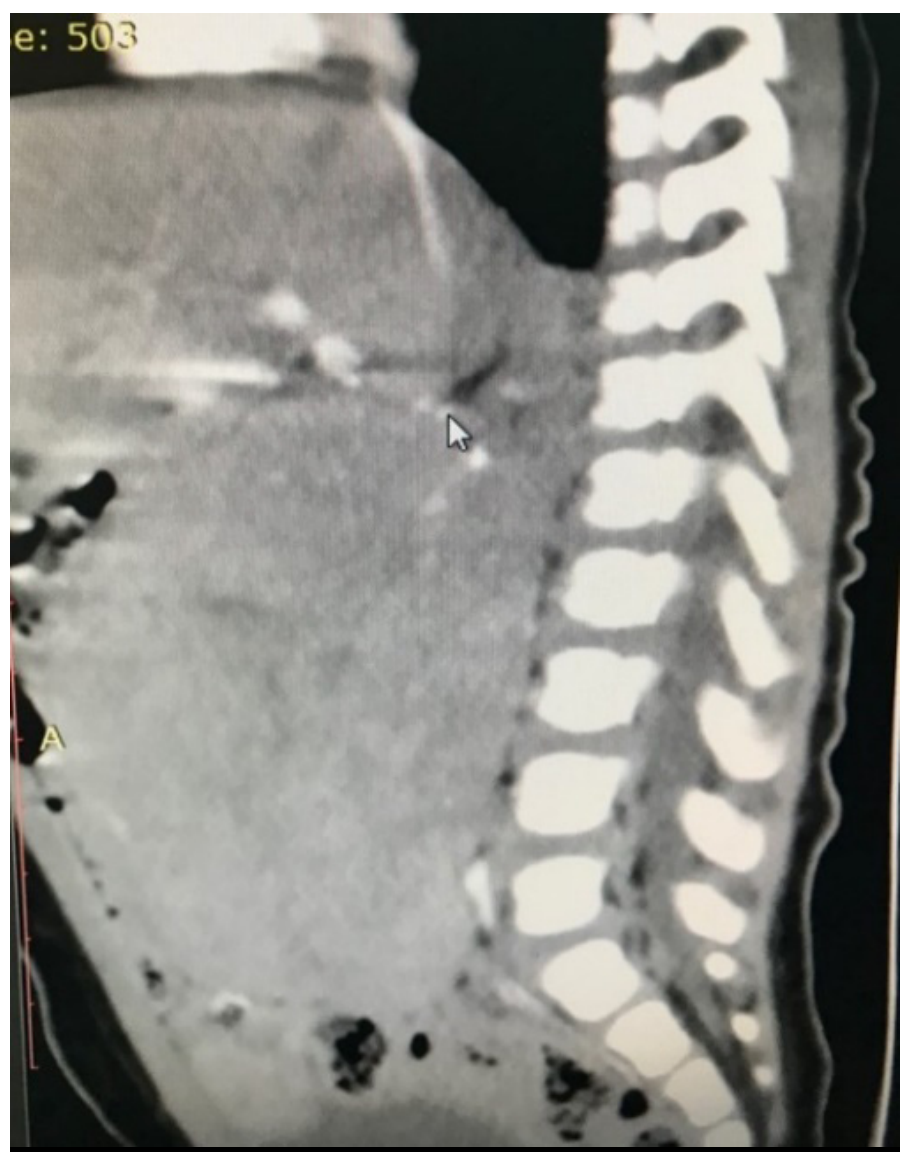

Fig. 1. Imaging studies performed on admission. Enhanced computed tomography revealed a large mass (Arrow 8.3*8.7 cm) in the retroperitoneal cavity at the sub hepatic region with multiple internal foci of calcification.

\section{Discussion}

Neuroblastoma is a solid malignant tumor typically seen in children, but rare adult onset cases exist [4].

Among pediatric solid malignancies, neuroblastomas are well known to extend in many directions. In a review of literature, it is found that many case reports clearly describing pediatric abdominal neuroblastomas with extension into the vessels. Many of these cases were managed with initial chemotherapy but there is a lot of cases that underwent operation directly [2], even laparotomy or open operation. Although the use of a laparoscope inresection is still challenging and has many limitations [3].

Our patient did not have intraoperative or postoperative complications and was discharged five days after surgery. The patient resumed all activities within one week after the surgery, and no signs of recurrence were found during the regular postoperative follow-ups. This case shows that treatment without chemotherapy prior operation cannot only successfully yield the same oncological results but also help reduce the disease recurrence rate and shorten the durations of surgery, hospitalization stay, and postoperative recovery.

Although initial chemotherapy is usually started with knowledge of the results of biopsies, it was difficult to determine the timing to start chemotherapy in our case. We considered the patient's condition to be an oncologic emergency due to the presence of the tumor extended to vessels and chemotherapy was unavailable. So the primary tumor was removed according to the American Pediatric Surgical Association and also by consideration of the tumor extension into adjacent major blood vessels, risk of hemorrhage, and the patient's overall tumor burden [5].

The study by Castel et al. denied the therapeutic effect of surgical resection of high stage and suggested that age, N-myc gene amplification, and distant metastasis have a much greater influence on the prognosis than complete resection. Therefore, the treatment should be a combination of surgery, radiotherapy, chemotherapy, and other treatments. Preoperative chemotherapy is necessary to remove the NB that envelopes important peritoneal blood vessels. Through chemotherapy, tumors can shrink significantly and harden, and the blood supply and other risk factors can decrease, making the resection of unresectable tumors posible [3].

Surgeons mainly remove the entire tumor but even if some of the tumor is left behind, it doesn't always mean that the tumor will come back irrespective of whether chemotherapy and other treatments is given after surgery [6]. 
Children with neuroblastoma will get chemotherapy depends on their risk group. Some children with neuroblastoma are treated with chemo either before surgery (neoadjuvant chemotherapy) or after surgery (adjuvant chemotherapy). It is evident that for larger, localized tumors surgical resection is pursued but for children who show symptoms, limited chemotherapy is given without surgical palliation or radiation therapy [6].

Treatment for neuroblastoma varies significantly based on the risk for relapse. Patients with disease classified as low risk have tumors that are localized to one área. These can be mostly or completely removed by a surgeon or if features indicate that the tumor is unlikely to spread or come back , surgical removal of the tumor may be necessary for children [5].

Doctors give chemo in cycles, which consist of treatment on a few days in a row, followed by time off to allow the body to recover. The cycles are typically repeated every 3 or 4 weeks. The total length of treatment depends on the risk group of the child. Usually, higher risk groups require longer treatment [6].

\section{Conclusion}

The goal in the abdominal Primaries is the most complete resection possible without putting organs at risk. Tumor masses encasing major visceral vessels that are not easily dissectible should be left in place. The major vessels include the celiac axis, superior mesenteric artery and vein, renal arteries and veins, and the inferior mesenteric artery. At no point, the surgeon should risk kidney loss, or bowel infarction in resection of non-high-risk abdominal neuroblastoma.

\section{References}

1. Starchenko II, Dyachenko L V., Prylutskyi OK, Vynnyk NI, Filenko BM. The observation of congenital retroperitoneal large size neuroblastoma. Exp Oncol. 2019;41(2):179-181. doi: 10.32471/ exp-oncology.2312-8852. vol-41-no-2.13321

2. Uehara S, Takama Y, Yoshida H, Hashii Y, Oue T, Usui N. Abdominal neuroblastoma with inferior vena cava and right atrial extension. J Pediatr Surg Case Reports. 2013;1(6):119-121. doi: 10.1016/j. epsc.2013.05.001

3. Chen DX, Hou YH, Jiang YN, Shao LW, Wang SJ, Wang XQ. Removal of pediatric stage IV neuroblastoma by robot-assisted laparoscopy: A case report and literature review. World J Clin Cases. 2019;7(12):1499-1507. doi:10.12998/wjcc. v7.i12.1499

4. Watanabe S, Hara F, Kondo Y, et al. A Case of Retroperitoneal Primary Neuroblastoma that Developed in Adulthood. J Tumour Res Reports. 2018;03(01):1-5.doi: 10.35248/26841614.18.3.116

5. Committee C. Handbook for Children with Neuroblastoma. Published online 2015.

6. Kendall-Raynor P. Treating neuroblastoma. Cancer Pract. 2018;17(5):10-10. doi:10.7748/ cnp.17.5.10.s8

7. American Cancer Society's (ACS) publication, Cancer Facts \& Figures 2020 